H. Ohashi

Nagoya Math. J.

Vol. 195 (2009), 165-186

\title{
ENRIQUES SURFACES COVERED BY JACOBIAN KUMMER SURFACES
}

\author{
HISANORI OHASHI
}

\begin{abstract}
This paper classifies Enriques surfaces whose $K 3$-cover is a fixed Picard-general Jacobian Kummer surface. There are exactly 31 such surfaces. We describe the free involutions which give these Enriques surfaces explicitly. As a biproduct, we show that $\operatorname{Aut}(X)$ is generated by elements of order 2 , which is an improvement of the theorem of S. Kondo.
\end{abstract}

\section{$\S 1$. Introduction}

A $K 3$ surface is a simply connected compact complex surface whose canonical bundle is trivial. Every Enriques surface appears as a quotient of a $K 3$ surface by a fixed-point-free (shortly, free) involution. Theoretically, to consider an Enriques surface is equivalent to consider the pair of the covering $K 3$ surface and the free involution. For example, the period map for Enriques surfaces is constructed under this description. But the properties of free involutions on a fixed $K 3$ surface are rather unclear to us. The existence is already a special property, their geometric realizations and the isomorphism classes of the quotient Enriques surfaces are other problems.

For a fixed $K 3$ surface $X$, two quotient Enriques surfaces are isomorphic if and only if the two free involutions are conjugate in $\operatorname{Aut}(X)$. In [16] it is shown that the number of the conjugacy classes of free involutions (and more generally, of finite subgroups) are finite. There this number, i.e., the number of isomorphism classes of quotient Enriques surfaces, is computed for $K 3$ surfaces with Picard number $\rho=11$ or for Kummer surfaces associated with the product of two elliptic curves whose periods are very general.

The aim of this paper is to study fixed-point-free involutions on surfaces studied in [11], [12]. Let $C$ be a smooth projective curve of genus 2. Its Jacobian variety $J(C)$ is the abelian surface parametrizing divisor classes on $C$ of degree 0 . The quotient surface $J(C) /\left\{ \pm 1_{J(C)}\right\}$ has 16 nodes and

Received August 1, 2008.

Accepted February 7, 2009.

2000 Mathematics Subject Classification: 14J28. 
can be embedded into $\mathbb{P}^{3}$ as a quartic hypersurface. We call it the Kummer quartic surface associated with $C$ and denote by $\overline{K m}(J(C))=: \bar{X}$. The minimal desingularization $K m(J(C))=: X$ of $\overline{K m}(J(C))$ is called the $J a$ cobian Kummer surface associated with $C$, which is a $K 3$ surface. $X$ is Picard-general if the Picard number of $X$ equals 17 , the minimum possible value. In what follows, $X$ will always be a Picard-general Jacobian Kummer surface except for Sections 2 and 4.

In [13], Mukai observed that there exist three kinds of free involutions on $X$.

- A switch associated with an even theta characteristic $\beta$.

- A Hutchinson-Göpel (shortly HG) involution associated with a Göpel tetrad G.

- A Hutchinson-Weber (shortly HW) involution associated with a Weber hexad $W$.

Essentially these automorphisms date back more than a century, but their freeness are found only recently in comparison. Mukai studied HG involutions in connection with the numerically reflective involutions of Enriques surfaces. Also he conjectured that these are the all free involutions on $X$. In this paper we prove the following theorem and confirm the conjecture.

TheOREM 1.1. On a Picard-general Jacobian Kummer surface $X$, there are exactly $31=10+15+6$ free involutions up to conjugacy in $\operatorname{Aut}(X)$. 10 are switches, 15 are $H G$ involutions and 6 are $H W$ involutions.

In [12], Kondo proved that $\operatorname{Aut}(X)$ is generated by 32 translations and switches, 32 projections and correlations, 60 HG involutions, and 192 Keum's automorphisms. One point of the proof was that 192 Keum's automorphisms did not correspond in one-to-one way to the 192 facets of the polyhedral cone introduced by Borcherds and Kondo. Moreover they had infinite order while the others had order 2. In this respect, it can be expected that there exist 192 involutions which correspond in one-to-one way to the 192 facets of the polyhedral cone and together with the $32+32+60$ involutions they generate $\operatorname{Aut}(X)$. In fact, the $\mathrm{HW}$ involutions work well.

THEOREM 1.2. $\operatorname{Aut}(X)$ is generated by the following involutions: translations, switches, projections, correlations, $H G$ involutions and $H W$ involutions. 
This is a biproduct of the proof of Theorem 1.1.

The proof of Theorem 1.1 is given in the following way. In Section 2 we introduce an invariant of a free involution, called a patching subgroup, which is a subgroup of $A_{N S(X)}=N S(X)^{*} / N S(X)$. This subgroup appears naturally in the light of Nikulin's theory of lattices [15]. Under some condition, we can show the invariance of the patching subgroup under conjugations. Section 3, Proposition 3.4 shows conversely two free involutions are conjugate if their patching subgroups are the same, when $X$ is a Picard-general Jacobian Kummer surface. Simultaneously we see that $X$ has no more than 31 non-isomorphic Enriques quotients. These two Sections reduce the proof of Theorem 1.1 to concrete computations of patching subgroups of free involutions itemized above. The occurence of 31 distinct patching subgroups shows Theorem 1.1. The computations are worked out in Sections 5-7. The result shows that the generators of patching subgroups are expressed in terms of the classical notions. It is summarized as follows.

In the switch case, let $\beta$ be an even theta characteristic and $\sigma_{\beta}$ be the switch. $\beta$ corresponds to a pair of Rosenhain subgroups $R_{1}, R_{2}$. Then the patching subgroup $\Gamma_{\sigma_{\beta}}$ is cyclic of order 4 and generated by

$$
H / 4+\sum_{\alpha \in R_{1}} N_{\alpha} / 2 .
$$

Of course we obtain the same group after replacing $R_{1}$ by $R_{2}$ in this case.

In the HG involution case, let $G$ be a Göpel tetrad and $\sigma_{G}$ be the HG involution. Then the patching subgroup $\Gamma_{\sigma_{G}}$ is 2-elementary abelian of order 4 and generated by

$$
H / 2 \text { and } \sum_{\alpha \in G} N_{\alpha} / 2 .
$$

We remark that this result of $\mathrm{HG}$ involution case also follows from the computations of [13].

In the HW involution case, let $W$ be a Weber hexad and $\sigma_{W}$ be the HW involution. Then the patching subgroup $\Gamma_{\sigma_{W}}$ is cyclic of order 4 and generated by

$$
H / 4+\sum_{\alpha \in W} N_{\alpha} / 2 .
$$

The divisors $H, N_{\alpha} \in N S(X)$ and also the classical notions appeared here will be defined in Section 4, where we recall the basic properties of Jacobian Kummer surfaces. After fixing the basis of $A_{N S(X)}$, we can easily check that there appear 31 distinct patching subgroups. 
Notation. We refer the readers to [15] for the basic properties of the finite quadratic form $\left(A_{L}, b_{L}, q_{L}\right)$ associated with an even nondegenerate lattice $L$. By definition, $A_{L}$ is the finite abelian group $L^{*} / L, b_{L}: A_{L} \times A_{L} \rightarrow$ $\mathbb{Q} / \mathbb{Z}$ is the symmetric bilinear form and $q_{L}: A_{L} \rightarrow \mathbb{Q} / 2 \mathbb{Z}$ is the quadratic form, both naturally induced from that of $L$. Usually we denote finite forms by $\left(A_{L}, q_{L}\right)$, omitting $b_{L}$, or only by $A_{L}$.

The hyperbolic plane is denoted by $U$, the root lattices $A_{l}, D_{m}, E_{n}$ are considered to be negative definite. The rank one lattice $\langle 2 n\rangle$ is also used in this paper. On finite forms, $u(2)$ is the associated form of the lattice $U(2)$, $\langle 1 / 2 n\rangle$ is that of $\langle 2 n\rangle$. The set of generators $\{e, f\}$ of $u(2)$ satisfying

$$
q(e)=q(f)=0, \quad b(e, f)=1 / 2
$$

is called the standard generator.

For a lattice $T$ and $k=\mathbb{Q}, \mathbb{C}$ we denote the scalar extension by $T_{k}$. If $T$ is a lattice and $T_{\mathbb{C}}$ is equipped with a Hodge structure, then $\operatorname{Aut}_{\text {Hodge }}(T)$ is a subgroup of $O(T)$ whose elements preserve the Hodge decomposition.

\section{$\S 2$. The method of counting}

In this section $X$ is any $K 3$ surface. Let $\sigma$ be a free involution on $X$. The (-1)-eigenspace of the action of $\sigma$ on $N S(X)$ is denoted by $K$. Then it is well-known that $K$ is negative definite, contains no $(-2)$-element and the primitive hull of $K \oplus T_{X}$ in $H^{2}(X, \mathbb{Z})$ is isometric to $U \oplus U(2) \oplus E_{8}(2)=: N$. We choose a marking $\phi: \overline{K \oplus T_{X}} \rightarrow N$ for this isometry.

The nonzero global holomorphic 2 -form $\omega_{X}$ on $X$ determines via $\phi$ a point in $\mathcal{D}(N) / O(N)$, which is the period of the Enriques surface $Y:=X / \sigma$, where

$$
\mathcal{D}(N):=\left\{\mathbb{C} \omega \in \mathbb{P}\left(N_{\mathbb{C}}\right) \mid \omega \in N \otimes \mathbb{C}, \omega \cdot \omega=0, \omega \cdot \bar{\omega}>0\right\}
$$

is the (two copies of) bounded symmetric domain of type IV associated to lattice $N$ of signature $(2,10)$. Obviously this period is independent of the choice of $\phi$ and the Torelli theorem of Enriques surfaces says that this point determines the isomorphism class of $Y$ uniquely.

Conversely given a primitive embedding $\phi: T_{X} \rightarrow N$ such that the orthogonal complement $K$ is free from $(-2)$-elements, by the surjectivity there exists an Enriques surface $Y$ whose period is exactly $\left[\phi\left(\mathbb{C} \omega_{X}\right)\right]$. If $\rho(X) \geq 12$ then [10, Theorem 1$]$ shows that $X$ is isomorphic to the universal double cover of $Y$. Even if $\rho(X) \leq 11$ the same holds, whose proof is in [17].

Thus we have shown 
Proposition 2.1. There is a one-to-one correspondence between the sets

$$
\{\text { Enriques quotients of } X\} /(\text { isomorphisms })
$$

and

$$
\left\{\begin{array}{l}
\text { Primitive embeddings } \phi: T_{X} \rightarrow N \\
\text { such that } K=T_{X}^{\perp} \text { contains } \\
\text { no }(-2) \text {-elements }
\end{array}\right\} /(\text { Hodge isometries of } N),
$$

where for each $\phi$ we equip $N$ with a Hodge structure induced from that of $T_{X}$ by $\phi$.

In the following, we identify $\overline{K \oplus T_{X}}$ with $N$ by $\phi$. By [15], there are subgroups $\Gamma_{K} \subset A_{K}$ and $\Gamma_{T_{X}} \subset A_{T_{X}}$ and a sign-reversing isometry $\varphi: \Gamma_{K} \stackrel{\sim}{\rightarrow} \Gamma_{T_{X}}$ such that $N$ is the sublattice of $K_{\mathbb{Q}} \oplus T_{X, \mathbb{Q}}$ generated by $K$, $T_{X}$ and $\left\{(x, \varphi(x)) \mid x \in \Gamma_{K}\right\}$.

Definition 2.2. The patching subgroup $\Gamma_{\sigma}$ of the free involution $\sigma$ is the inverse image of $\Gamma_{T_{X}}$ by the natural sign-reversing isometry $A_{N S(X)} \stackrel{\sim}{\rightarrow}$ $A_{T_{X}}$.

Under a condition, $\Gamma_{\sigma}$ is an invariant of a conjugacy class which is very computable.

Proposition 2.3. If $\operatorname{Aut}_{\text {Hodge }}\left(T_{X}\right)=\{ \pm \mathrm{id}\}$, then $\Gamma_{\sigma}$ depends only on the isomorphism class of the quotient Enriques surface.

Proof. By Proposition 2.1, conjugate free involutions induce on $N$ an isometric Hodge structure. Any Hodge isometry of $N$ preserves $K=\omega_{X}^{\perp}$ and hence $T_{X}$. Thus it induces \pm id on $T_{X}$ and preserves the subgroup $\Gamma_{T_{X}}$.

Remark 2.4. The condition above is weak. It is true if $\rho(X)$ is odd, see $[12$, p. 597], or even if $\rho(X)$ is even, it is true if $X$ is very general in the period domain ([16, Proposition 3.1]).

In general there are free involutions not conjugate each other but with the same $\Gamma_{\sigma}$. However in the Picard-general Jacobian Kummer case, $\Gamma_{\sigma}$ completely classifies free involutions. This will be shown in the next section.

The computation of $\Gamma_{\sigma}$ is done by 
Lemma 2.5. Let $\sigma, K$ as above. Then

$$
\Gamma_{\sigma}=\left\{[x] \in N S(X)^{*} / N S(X) \mid \exists[y] \in K^{*} / K, x-y \in N S(X)\right\} .
$$

Proof. Let $\rho: A_{N S(X)} \rightarrow A_{T_{X}}$ be the canonical isomorphism. Then $\rho([x])=[z]$ is equivalent to $x+z \in H^{2}(X, \mathbb{Z})$. Since $\Gamma_{T_{X}}=\left\{[z] \in T_{X}^{*} / T_{X}\right.$ $\left.\exists[y] \in K^{*} / K, y+z \in N\right\}$,

$$
\begin{aligned}
\Gamma_{\sigma} & =\left\{[x] \in N S(X)^{*} / N S(X) \mid \rho([x]) \in \Gamma_{T_{X}}\right\} \\
& =\left\{[x] \in N S(X)^{*} / N S(X) \mid \exists[y] \in K^{*} / K, x-y \in N S(X)\right\} .
\end{aligned}
$$

This is what we need.

\section{$\S 3 . \quad$ Invariants of free involutions}

Let $C$ be a genus 2 curve, $J(C)$ its Jacobian and $K m(J(C))=X$ the associated Jacobian Kummer surface as in the Introduction. As is wellknown, $J(C)$ contains $C$ as a theta divisor:

$$
\Theta=\left\{\left[p-p_{0}\right] \mid p \in C\right\} \subset J(C), \quad p_{0} \in C .
$$

Hence $\operatorname{rank} N S(J(C)) \geq 1$ and $\operatorname{rank} N S(X) \geq 17$ holds. When we have the equality, we call $X$ Picard-general. In this case, since $T_{J(C)}=U^{\oplus 2} \oplus\langle-2\rangle$ we have $T_{X}=U(2)^{\oplus 2} \oplus\langle-4\rangle$ and $N S(X)=U \oplus D_{4}^{\oplus 2} \oplus D_{7}$.

For simplicity, we put $T:=T_{X}$. Suppose we are given a primitive embedding of $T$ into $N$ such that the orthogonal complement is free from (-2)-elements, as in Proposition 2.1. First we determine the orthogonal complement.

Proposition 3.1. The lattice $K=T_{X}^{\perp}$ is isometric to $E_{7}(2)$.

Proof. Consider the unique embedding of $N$ into the abstract $K 3$ lattice $L$. The orthogonal complement is denoted by $M, M \simeq U(2) \oplus E_{8}(2)$. By [15], we have the following isomorphism of discriminant quadratic forms:

$$
-q_{K} \simeq\left(\left.q_{M} \oplus q_{T}\right|_{\Gamma^{\perp}}\right) / \Gamma
$$

where $\Gamma$ is the pushout (i.e. the graph) of a sign-reversing isometry of subgroups $\Gamma_{M} \subset A_{M}$ and $\Gamma_{T} \subset A_{T}$.

For a finite quadratic form $(A, q)$, we denote the quadratic form induced on the 2-torsion subgroup $A_{2}=\{x \in A \mid 2 x=0\}$ by $\left(A_{2}, q_{2}\right)$. Note that even if $q$ is nondegenerate, $q_{2}$ may be degenerate. 
In our equality (3.1), $A_{M}$ is 2-elementary, hence $\Gamma$ is 2-elementary and $\Gamma_{T}$ is contained in $\left(A_{T}\right)_{2}$. Put $\# \Gamma=2^{a}$. This shows $a \leq 5=l_{2}\left(A_{T}\right)$, where $l_{2}$ denotes the number of minimal generators of the 2-Sylow subgroup of $A_{T}$.

Also it follows

$$
\left(\left.\left(A_{M} \oplus A_{T}\right)_{2}\right|_{\Gamma^{\perp}}\right) / \Gamma \subset\left(\left.A_{M} \oplus A_{T}\right|_{\Gamma^{\perp}}\right) / \Gamma=A_{K},
$$

since $\Gamma$ is 2-elementary. $\left(A_{M} \oplus A_{T}\right)_{2}$ has a radical of order 2 contained in $\left(A_{T}\right)_{2}$. Since $\Gamma$ is a graph, this radical is not contained in $\Gamma$. This shows that $\#\left(\left.\left(A_{M} \oplus A_{T}\right)_{2}\right|_{\Gamma^{\perp}}\right)=2^{15-a}$. Thus the order of the left-hand-side of (3.2) is $2^{15-2 a}$. Since $K$ is of rank 7 , we have $15-2 a \leq 7$ and hence $a=4,5$.

We show that if $a=5$ then $K$ contains $(-2)$-elements and contradicts the assumption. For this, first note that in this case $\Gamma_{T}=\left(A_{T}\right)_{2}$ is uniquely determined and the embedding of $\Gamma_{T}$ in $A_{M} \simeq u(2)^{\oplus 5}$ is unique up to isomorphism by Witt's theorem. So we can compute $q_{K}$ directly and get $q_{K} \simeq u(2)^{\oplus 2} \oplus\langle 1 / 4\rangle$. From this we see that there are inclusions $K \subset K^{\prime} \subset$ $\langle-1\rangle^{\oplus 7}$ such that $K^{\prime}$ is an even lattice, $\left[K^{\prime}: K\right]=4$ and $\left[\langle-1\rangle^{\oplus 7}: K^{\prime}\right]=2$. By the definition of $D_{7}, K^{\prime} \simeq D_{7}$. Consider the Dynkin diagram of $D_{7}$ and take a subgraph isomorphic to $A_{6}$ with vertices $e_{1}, \ldots, e_{6}$ in this order. Put $f_{0}=0, f_{j}=e_{1}+\cdots+e_{j}, 1 \leq j \leq 6$. Any difference of two of these seven elements have self-intersection $(-2)$. If $K$ has no $(-2)$-elements, then $\left\{f_{j}\right\}_{0 \leq j \leq 6}$ cannot be in the same residue class of $K^{\prime} / K$. Then we must have $\left[K^{\prime}: K\right] \geq 7$ and contradiction.

Thus we obtain $a=4$. From (3.2), we see that $\#\left(A_{K}\right)_{2} \geq 2^{7}$. It follows that $K(1 / 2)$ is an integral (may be odd) lattice and $\operatorname{det} K(1 / 2)=-2$. By assumption, the minimal norm of the positive definite lattice $K(-1 / 2)$ is greater than 1. It follows from [3, p. 400, Table 15.8] that $K(1 / 2) \simeq$ $\left(\langle-2\rangle^{\perp}\right.$ in $\left.E_{8}\right) \simeq E_{7}$.

The following nature of the lattice $K=E_{7}(2)$ will be used.

LEMma 3.2. The canonical homomorphism $\sigma: O(K) \rightarrow O\left(q_{K}\right)$ is surjective.

Proof. The same property for the lattice $E_{8}(2)$ is known by [1]. We reduce the lemma to this case. Firstly, we know the orders of the two groups. By [2], $\# O\left(E_{7}(2)\right)=\# O\left(E_{7}\right)=2^{10} \cdot 3^{4} \cdot 5 \cdot 7$. On the other hand, we can easily compute the order of $O\left(q_{K}\right)$ as $\# O\left(q_{K}\right)=\# O\left(u(2)^{\oplus 2} \oplus\langle 1 / 4\rangle\right)=$ $2^{10} \cdot 3^{4} \cdot 5 \cdot 7$ (c.f. Lemma 3.3). Thus it is enough to show that $\sigma$ is injective. 
We take a (-4)-element $r$ of $E_{8}(2)$ and identify $K$ with $r^{\perp}$. Obviously $g \in \operatorname{ker} \sigma$ can be extended to an isometry $\bar{g}$ of $E_{8}(2)$ by defining $\bar{g}(r)=r$. It is clear that $\bar{g}$ acts on the discriminant $A_{E_{8}(2)}$ trivially. It follows from $[1$, Proposition 1.7] that $\bar{g}= \pm \mathrm{id}$. Since $\bar{g}(r)=r, \bar{g}=\mathrm{id}$.

Because $\left(A_{K}, q_{K}\right) \simeq u(2)^{\oplus 3} \oplus\langle 1 / 4\rangle$, the next lemma is also used.

Lemma 3.3. Let $(A, q)=u(2)^{m} \oplus\langle 1 / 4\rangle$ be a finite quadratic form. Then the action of $O(q)$ on $A$ decomposes $A$ into 6 orbits. If we denote the standard generator of one of $u(2)$ by $\{e, f\}$ and that of $\langle 1 / 4\rangle$ by $\{g\}$, they are as in the following table.

\begin{tabular}{lll}
\hline a representative & length & square \\
\hline 0 & 1 & 0 \\
$2 g$ & 1 & 1 \\
$e$ & $2^{2 m}-1$ & 0 \\
$e+f$ & $2^{2 m}-1$ & 1 \\
$g$ & $2^{2 m}+2^{m}$ & $1 / 4$ \\
$e+f+g$ & $2^{2 m}-2^{m}$ & $-3 / 4$ \\
\hline
\end{tabular}

In this table, for a representative $x$, the length is $\#(O(q) \cdot x)$ and the square is $q(x) \in \mathbb{Q} / 2 \mathbb{Z}$.

The proof is given by induction on $m$ and we omit it.

Now we are going to describe the latter set of Proposition 2.1, i.e., we classify the Hodge structures on $N$ induced from embeddings $T \subset N$ as in the proposition. We recall Definition 2.2, there is an isomorphism

$$
\left.q_{N} \simeq\left(q_{K} \oplus q_{T}\right)\right|_{\Gamma^{\perp}} / \Gamma
$$

where $\Gamma$ is the pushout of a sign-reversing isometry of subgroups $\Gamma_{K} \subset A_{K}$ and $\Gamma_{T} \subset A_{T}$. By Proposition 2.3, $\Gamma_{T}$ is an invariant of the Hodge structure.

We will prove the converse. Namely, suppose we have two embeddings $T \subset N_{1}$ and $T \subset N_{2}$ whose orthogonal complements are denoted by $K_{i}$. For each embedding we have $\left(\Gamma_{i}, \Gamma_{K, i} \Gamma_{T, i}\right)$ and the equality (3.3). What we want to show is

$$
(*): \Gamma_{T, 1}=\Gamma_{T, 2} \Longrightarrow N_{1} \simeq N_{2} \text { (Hodge isometry). }
$$


The argument goes as follows. Assume we could find an isometry $\sigma_{K}$ : $A_{K_{1}} \rightarrow A_{K_{2}}$ such that the following commutes.

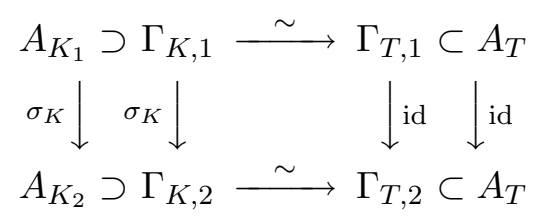

Then by Lemma 3.2 we can lift $\sigma_{K}$ to $\sigma_{K}^{\prime}: K_{1} \stackrel{\sim}{\rightarrow} K_{2}$ and the pair $\left(\sigma_{K}^{\prime}, \operatorname{id}_{T}\right)$ can be lifted to an Hodge isometry $N_{1} \stackrel{\sim}{\rightarrow} N_{2}$. Thus it is enough to find $\sigma_{K}$.

By Proposition 3.1, $[N: K \oplus T]=\# \Gamma=4$. Thus there are two possibilities of underlying groups of $\Gamma_{K} \simeq \Gamma_{T}$. We consider each case separately.

First we consider the case $\Gamma_{T, i} \simeq \mathbb{Z} / 4 \mathbb{Z}$. The square of the generator $g_{T} \in \Gamma_{T, 1}=\Gamma_{T, 2}$ is independent of the choice and there are two possibilities, $q_{T}\left(g_{T}\right)=-1 / 4$ or $3 / 4$. Let $\left(g_{K, i}, g_{T}\right) \in \Gamma_{i}$. We have $q_{K_{i}}\left(g_{K, i}\right)=1 / 4$ or $-3 / 4$ respectively. By Lemma 3.3, in these cases we can find $\sigma_{K}$ and $(*)$ is proved. We find easily that there are 10 subgroups $\Gamma_{T}$ satisfying $q_{T}\left(g_{T}\right)=-1 / 4$. Also there are 6 with $q_{T}\left(g_{T}\right)=3 / 4$.

Second we consider the case $\Gamma_{T} \simeq \mathbb{Z} / 2 \mathbb{Z} \oplus \mathbb{Z} / 2 \mathbb{Z}$. The argument becomes slightly complicated, but the conclusion is the same. To prove $(*)$ in this case, first we show that $\Gamma_{i}$ always contains a particular element. Here, for a clear argument, we take generators $g_{i}$ and $g^{\prime}$ of $\langle 1 / 4\rangle \subset A_{K_{i}}$ and $\langle-1 / 4\rangle \subset A_{T}$ respectively. We denote an element of $A_{K_{i}} \oplus A_{T}$ by

$$
(x, y ; z, w) \in A_{K_{i}} \oplus A_{T} ; x \in u(2)^{\oplus 3}, y \in\langle 1 / 4\rangle, z \in u(2)^{\oplus 2}, w \in\langle-1 / 4\rangle .
$$

Then the claim is that

$$
\left(0,2 g_{i} ; 0,2 g^{\prime}\right) \in \Gamma_{i} .
$$

In fact, since $\Gamma_{i}$ is contained in $\left(A_{K_{i}} \oplus A_{T}\right)_{2}$, the radical element $\left(0,2 g_{i} ; 0,0\right)$ of $\left(A_{K_{i}} \oplus A_{T}\right)_{2}$ is in $\Gamma_{i}^{\perp}$. Hence its residue class $\left(0,2 g_{i} ; 0,0\right)+\Gamma_{i}$ determines an element of $A_{N_{i}}$ by the isomorphism (3.3). It is nonzero because $q_{K_{i}}\left(2 g_{i}\right)=1$. Since $A_{N_{i}}$ is nondegenerate, there exists an element $(x, y ; z, w)+\Gamma_{i} \in A_{N_{i}}$ with $\left(0,2 g_{i} ; 0,0\right) \cdot(x, y ; z, w)=1 / 2$. It follows $y= \pm g_{i}$. Further since $\left(q_{K_{i}} \oplus q_{T}\right)(x, y ; z, w) \in \mathbb{Z}$, it follows $w= \pm g^{\prime}$, i.e., there exists an element in $\Gamma_{i}^{\perp}$ of the form $\left(x, \pm g_{i} ; z, \pm g^{\prime}\right)$. Since the residue class of this element is of order 2 in $A_{N_{i}}$, we have that $\left(0,2 g_{i} ; 0,2 g^{\prime}\right) \in \Gamma_{i}$.

Let $\Gamma_{T, 1}=\Gamma_{T, 2}=\left\langle 2 g^{\prime}, \alpha\right\rangle$. Replacing $\alpha$ by $\alpha+2 g^{\prime}$ if necessary, we can assume $q_{T}(\alpha)=0$. Let $\left(\beta_{i}, \alpha\right) \in \Gamma_{i}, q_{K_{i}}\left(\beta_{i}\right)=0$. By Lemma 3.3, we can 
find $\sigma_{K}: A_{K_{1}} \stackrel{\sim}{\rightarrow} A_{K_{2}}$ which takes $\beta_{1}$ to $\beta_{2}$. This $\sigma_{K}$ must take $2 g_{1}$ to $2 g_{2}$, so we have now proved $(*)$. There are 15 possible $\Gamma_{T}$ in this case.

In summary, we have obtained the following.

Proposition 3.4. Let $X$ be a Picard-general Jacobian Kummer surface. Then free involutions $\sigma_{1}, \sigma_{2}$ are conjugate if and only if the patching subgroups $\Gamma_{\sigma_{1}}, \Gamma_{\sigma_{2}}$ coincide. There exist (at most) $31=10+15+6$ free involutions.

The existence of 31 free involutions is assured by concrete constructions in the following sections.

\section{§4. The $(16)_{6}$ configuration on a Jacobian Kummer surface}

In this section we recall and prepare notations concerning the divisors on Jacobian Kummer surfaces. The content of this section is known, references are [11], [12], [4].

The index set. Let $C$ be a smooth projective curve of genus 2 . It is a double cover of $\mathbb{P}^{1}$ which ramifies at 6 Weierstrass points $\left\{p_{1}, \ldots, p_{6}\right\} \subset C$. Here we should notice the linear equivalence

$$
p_{i}+p_{j}+p_{k}-p_{l}-p_{m}-p_{n} \sim 0
$$

for an arbitrary permutation $\{i, j, k, l, m, n\}$ of $\{1, \ldots, 6\}$. The set of theta characteristics of $C$ is by definition

$$
S(C)=\left\{D \in \operatorname{Pic}(C) \mid 2 D \sim K_{C}\right\} .
$$

They are divided into odd theta characteristics $\left\{\left[p_{i}\right] \mid i=1, \ldots, 6\right\}$ and even ones $\left\{\left[p_{i}+p_{j}-p_{k}\right] \mid i, j, k\right.$ are distinct each other $\}$. There are 16 theta characteristics.

The Jacobian variety $J(C)$ consists of divisor classes of degree 0 on $C$. We denote by $J(C)_{2}$ the set of sixteen 2-torsion points of $J(C)$. Then

$$
J(C)_{2}=\{0\} \cup\left\{\left[p_{i}-p_{j}\right] \mid i \neq j\right\} .
$$

These $16+16=32$ divisor classes naturally correspond to partitions of the set $\{1, \ldots, 6\}$ into two subsets in the following way.

$$
\begin{aligned}
{\left[p_{i}\right] \in S(C) } & \longleftrightarrow\{i\} \cup\{i\}^{c} . \\
{\left[p_{i}+p_{j}-p_{k}\right] \in S(C) } & \longleftrightarrow\{i, j, k\} \cup\{i, j, k\}^{c} . \\
{\left[p_{i}-p_{j}\right] \in J(C)_{2} } & \longleftrightarrow\{i, j\} \cup\{i, j\}^{c} . \\
0 \in J(C)_{2} & \longleftrightarrow \emptyset \cup\{1, \ldots, 6\},
\end{aligned}
$$


where the complement is taken in the set $\{1, \ldots, 6\}$. We denote these partitions by exhibiting one of the subsets, surrounded by [ ]. For example, $p_{1}-p_{2}$ corresponds to $[12]=[3456], p_{1}+p_{2}-p_{3}$ corresponds to $[123]=[456]$, etc. $[\emptyset]$ is denoted by $[0]$. In this notation, we see that the symmetric difference of subsets $\alpha, \beta$ of $\{1, \ldots, 6\}$ corresponds to addition or difference in $\operatorname{Div}(C)$ as follows.

$$
\begin{array}{ll}
{[\alpha \ominus \beta]=[\alpha]-[\beta]} & \text { if }[\alpha],[\beta] \in S(C), \\
{[\alpha \ominus \beta]=[\alpha]+[\beta]} & \text { otherwise. }
\end{array}
$$

When we use a partition $[\alpha]$ as an index, [ ] will be omitted.

The $(16)_{6}$ configuration. The sixteen theta divisors on $J(C)$ corresponding to $\beta \in S(C)$ are

$$
\Theta_{\beta}=\{[p-\beta] \in J(C) \mid p \in C\} .
$$

The sixteen nodes $\left\{n_{\alpha} \in \bar{X} \mid \alpha \in J(C)_{2}\right\}$ on $\bar{X}=J(C) /\{ \pm 1\}$ are the images of $\alpha \in J(C)_{2}$. On the minimal desingularization $X$ of $\bar{X}, n_{\alpha}$ is blown up to give a smooth rational curve $N_{\alpha}$ on $X$. The tropes $\overline{T_{\beta}} \subset \bar{X}$ and $T_{\beta} \subset X$ are the strict transforms of $\Theta_{\beta}$. Hence we obtain $32(-2)$-curves $\left\{N_{\alpha}, T_{\beta}\right\}_{\alpha, \beta}$ on $X$. The incidence relation between these divisors is called the $(16)_{6}$ configuration. It is given explicitly by

$$
\begin{gathered}
\left(N_{\alpha}, N_{\alpha^{\prime}}\right)=-2 \delta_{\alpha, \alpha^{\prime}}, \quad\left(T_{\beta}, T_{\beta^{\prime}}\right)=-2 \delta_{\beta, \beta^{\prime}}, \\
\left(N_{\alpha}, T_{\beta}\right)=1 \Longleftrightarrow \alpha+\beta \in\{[1],[2],[3],[4],[5],[6]\} .
\end{gathered}
$$

A permutation of the set $\left\{N_{\alpha}, T_{\beta}\right\}_{\alpha, \beta}$ which preserves the incidence relation above is called an automorphism. Nikulin [14] showed that the automorphism group is isomorphic to $(\mathbb{Z} / 2 \mathbb{Z})^{5} \rtimes \mathfrak{S}_{6}$, where $(\mathbb{Z} / 2 \mathbb{Z})^{5}$ consists of automorphisms induced from translations by elements of $J(C)_{2} \cup S(C)$ and $\mathfrak{S}_{6}$ acts on the index set $\{1, \ldots, 6\}$. We took our notations as above because this $\mathfrak{S}_{6}$-action is best seen.

Translations with respect to $\alpha \in J(C)_{2}$ are geometrically realized on $J(C)$. They induce automorphisms $t_{\alpha}$ of $X$. These are the translations in the classical terms. In the next section we will see that translations with respect to $\beta \in S(C)$ are also geometrically realized by $\sigma_{\beta} \in \operatorname{Aut}(X)$. These $\sigma_{\beta}$ are the switches. On the other hand, in general the action of $\mathfrak{S}_{6}$ cannot be lifted to an automorphism of $X$. 
Remark. In [11] (in the reference) and [12], the notations are a little different. To adjust notations of [12] to ours, first we regard $p_{0}$ of [12] as our $p_{6}$. Then the correspondence is as in below.

\begin{tabular}{c|cccccc}
{$[12]$} & $N_{0}$ & $N_{i}$ & $N_{i j}$ & $T_{0}$ & $T_{i}$ & $T_{i j}$ \\
\hline ours & $N_{0}$ & $N_{i 6}$ & $N_{i j}$ & $T_{6}$ & $T_{i}$ & $T_{i j 6}$
\end{tabular}

Lemma 4.1. ([12]) For $\beta \in S(C)$, let $\Lambda(\beta):=\left\{\alpha \in J(C)_{2} \mid\left(N_{\alpha}, T_{\beta}\right)=\right.$ $1\}$. Then the divisor class of

$$
H=2 T_{\beta}+\sum_{\alpha \in \Lambda(\beta)} N_{\alpha}
$$

is independent of $\beta$ and coincides with the pullback of the hyperplane section by the morphism $X \rightarrow \bar{X} \subset \mathbb{P}^{3}$.

Lemma 4.2. ([12]) Assume that $X$ is Picard-general.

1. $N S(X)$ is generated over $\mathbb{Z}$ by $\left\{N_{\alpha}, T_{\beta}\right\}_{\alpha, \beta}$.

2. $\left\{H, N_{\alpha}\right\}_{\alpha}$ is an orthogonal basis of $N S(X)_{\mathbb{Q}}$ over $\mathbb{Q}$.

3. A generator set of the discriminant group $A_{N S(X)}$ is given by

$$
\begin{gathered}
\mathrm{e}_{1}=\left(N_{26}+N_{12}+N_{36}+N_{13}\right) / 2, \quad \mathrm{f}_{1}=\left(N_{16}+N_{12}+N_{46}+N_{24}\right) / 2, \\
\mathrm{e}_{2}=\left(N_{26}+N_{12}+N_{46}+N_{14}\right) / 2, \quad \mathrm{f}_{2}=\left(N_{16}+N_{12}+N_{36}+N_{23}\right) / 2, \\
\mathrm{~g}=H / 4+\left(N_{0}+N_{16}+N_{26}+N_{12}\right) / 2 .
\end{gathered}
$$

Special subsets of nodes. Lastly we mention several special subsets of nodes of $\bar{X}$. See also [4]. We identify the set of nodes with $J(C)_{2}$ which is a 4 -dimensional vector space over $\mathbb{F}_{2}$. We have then the symplectic bilinear form

$$
\left([\alpha],\left[\alpha^{\prime}\right]\right) \longmapsto \#\left(\alpha \cap \alpha^{\prime}\right) \bmod 2,
$$

where we regard $\alpha, \alpha^{\prime}$ as subsets of $\{1, \ldots, 6\}$.

A 2-dimensional subspace $G$ is called Göpel if it is totally isotropic with respect to this bilinear form. The translations of Göpel subgroups are called Göpel tetrads. There are 60 Göpel tetrads. A 2-dimensional subspace $R$ which is not Göpel is called Rosenhain and its translations Rosenhain tetrads. There are 80 Rosenhain tetrads. A Weber hexad is a set with 6 elements which can be written as the symmetric difference of a Göpel tetrad 
and a Rosenhain tetrad. It can be shown that every Weber hexad is of one of the following forms

$$
\{0, i j, j k, k l, l m, m i\} \text { or }\{i j, j k, k i, i l, j m, k n\}
$$

according to whether it contains 0 or not. There are 192 Weber hexads.

In the following sections, we introduce automorphisms using these special subsets.

\section{§5. Switches}

Switches are one kind of automorphisms found by F. Klein [8]. The freeness in even cases is an easy consequence of the description of [9], although it is implicit there. Let $\beta \in S(C)$. For a smooth point $\bar{a} \in \bar{X}$, which means that the preimage of $\bar{a}$ in $J(C)$ is $\{a,-a\}$, the divisors $t_{a}\left(\Theta_{\beta}\right)$ and $t_{-a}\left(\Theta_{\beta}\right)$ intersect at two points, which is of the form

$$
t_{a}\left(\Theta_{\beta}\right) \cap t_{-a}\left(\Theta_{\beta}\right)=\{b,-b\} .
$$

The switch is defined by $\sigma_{\beta}: \bar{a} \mapsto \bar{b}$.

More theoretically, these switches are defined as the composite of the Gauss map

$$
G: \mathbb{P}^{3} \supset \bar{X} \rightarrow \bar{X}^{*} \subset\left(\mathbb{P}^{3}\right)^{*},
$$

which maps a smooth point $\bar{a}$ to $T_{\bar{a}} \bar{X}$, and the projective linear isomorphism

$$
F_{\beta}: \bar{X}^{*} \longrightarrow \bar{X}
$$

defined for each $\beta$. See [9].

$\sigma_{\beta}$ is a birational involution of $\bar{X}$. Hence it induces an involution of $X$, which we denote by the same $\sigma_{\beta}$. We can easily check that $\sigma_{\beta}$ interchanges $N_{\alpha}$ with $T_{\alpha+\beta}$ for $\forall \alpha \in J(C)_{2}$.

Proposition 5.1. For an even theta characteristic $\beta, \sigma_{\beta}$ is a free involution on $X$.

Proof. Suppose a smooth point $\bar{a} \in \bar{X}$ is a fixed point of $\sigma_{\beta}$. This is equivalent to $t_{a}\left(\Theta_{\beta}\right) \cap t_{-a}\left(\Theta_{\beta}\right)=\{a,-a\}$ and it is necessary that $a \in t_{a}\left(\Theta_{\beta}\right)$, $0 \in \Theta_{\beta}$. This is untrue if $\beta$ is even.

On the other hand, the divisor $N_{\alpha}$ is disjoint from $T_{\alpha+\beta}$, so $\sigma_{\beta}$ has no fixed points. 
Remark. (1) The proof above does not use the assumption of being Picard-general. Thus switches for even theta characteristics are always free involutions.

(2) The fixed point set of a switch for an odd theta characteristic is a curve of genus 5, named after Humbert.

Let $\sigma_{\beta}$ be a free switch. In the following computation, we take the case $\beta=[123]$ for simplicity. We can obtain the result for other cases by the action of $\mathfrak{S}_{6}$. Let $K$ be the $(-1)$-eigenspace of the action of $\sigma_{123}$ on $N S(X)$ as in Section 2.

Proposition 5.2. For Picard-general $X, K$ is generated over $\mathbb{Z}$ by the following elements.

$$
\begin{gathered}
f=N_{15}-T_{146}, \quad e_{2}=T_{145}-N_{16}, \quad e_{3}=N_{45}-T_{6}, \quad e_{4}=T_{123}-N_{0}, \\
e_{5}=N_{12}-T_{3}, \quad e_{6}=T_{124}-N_{34}, \quad e_{7}=N_{24}-T_{134}, \\
e_{1}=-(1 / 2)\left(f+2 e_{2}+3 e_{3}+4 e_{4}+3 e_{5}+2 e_{6}+e_{7}\right) .
\end{gathered}
$$

Proof. We can check that $\left\{f, e_{2}, \ldots, e_{7}\right\}$ spans a sublattice of $K$ isomorphic to $A_{7}(2)$. We now show $e_{1} \in N S(X)$. Modulo $N S(X)$,

$$
\begin{aligned}
e_{1} \equiv & \left(f+e_{3}+e_{5}+e_{7}\right) / 2 \\
\equiv & \left(N_{15}+N_{45}+N_{12}+N_{24}\right) / 2+\left(T_{146}+T_{6}+T_{3}+T_{134}\right) / 2 \\
= & \left(N_{15}+N_{45}+N_{12}+N_{24}\right) / 2 \\
& \quad+\left(\frac{H}{2} \cdot 4-\frac{1}{2} \sum_{\alpha \in \Lambda([146]) \cup \Lambda([6]) \cup \Lambda([3]) \cup \Lambda([134])} N_{\alpha}\right) / 2 \\
& \quad\left(N_{15}+N_{45}+N_{12}+N_{24}\right) / 2+H-\frac{1}{4} \sum_{\alpha \in J(C)_{2}-\{[15],[45],[12],[24]\}} 2 N_{\alpha} \\
\equiv & \sum_{\alpha \in J(C)_{2}} N_{\alpha} / 2 .
\end{aligned}
$$

The blow up $\widehat{J(C)}$ of $J(C)$ at points of $J(C)_{2}$ is the double cover of $X$ branched exactly over $\bigcup_{\alpha} N_{\alpha}$. Thus $e_{1} \in N S(X)$ follows.

Then it is easy to check that $\left\{e_{1}, e_{2}, \ldots, e_{7}\right\}$ spans a sublattice of $K$ isomorphic to $E_{7}(2)$. By Proposition 3.1, they coincide.

Proposition 5.3. The patching subgroup of $\sigma_{123}$ is the cyclic group generated by the element $\left[x=H / 4+\left(N_{0}+N_{12}+N_{23}+N_{31}\right) / 2\right] \in A_{N S(X)}$. 
Proof. The facts $x \in N S(X)^{*}$ and $y:=-\left(e_{1}+e_{5}+e_{7}\right) / 4+e_{5} / 2+e_{6} / 2 \in$ $K^{*}$ are easily checked. We use Lemma 2.5. We first check $x-y \in N S(X)$. This is because

$$
\begin{aligned}
y & =(1 / 8)\left(f+2 e_{2}+3 e_{3}+4 e_{4}+5 e_{5}+6 e_{6}-e_{7}\right) \\
& =H / 4-\left(N_{14}+N_{24}+N_{34}+N_{56}\right) / 2
\end{aligned}
$$

and

$$
\begin{aligned}
x-y & =(1 / 2)\left(N_{0}+N_{12}+N_{23}+N_{31}+N_{14}+N_{24}+N_{34}+N_{56}\right) \\
& \equiv T_{123}-T_{4} \equiv 0 .
\end{aligned}
$$

Thus $[x] \in \Gamma_{\sigma_{123}}$. Then since $[x]$ is of order 4 in $A_{N S(X)}$ and $\# \Gamma_{\sigma_{123}}=4$, $\Gamma_{\sigma_{123}}$ is generated by $[x]$.

OBSERVATiOn. In the expression of $[x],\left\{n_{0}, n_{12}, n_{23}, n_{31}\right\}$ is a Rosenhain subgroup defined in Section 4 . The class of $-x$ can be written as $\left[H / 4+\left(N_{0}+N_{45}+N_{56}+N_{64}\right) / 2\right]$, where $\left\{n_{0}, n_{45}, n_{56}, n_{64}\right\}$ is also a Rosenhain subgroup. In general, for an even theta characteristic $\beta$, the 6 -set $\Lambda(\beta)$ (see Lemma 4.1) can be uniquely written in the form $R_{1} \ominus R_{2}$ where $R_{i}$ are Rosenhain subgroups. In our case $\beta=[123], R_{1}=\left\{n_{0}, n_{12}, n_{23}, n_{31}\right\}$ and $R_{2}=\left\{n_{0}, n_{45}, n_{56}, n_{64}\right\}$.

Proposition 5.4. The patching subgroup of $\sigma_{\beta}$ for general $\beta$ is generated by $\left[H / 4+\left(\sum_{\alpha \in R} N_{\alpha}\right) / 2\right]$ where $R$ is one of the two Rosenhain subgroups corresponding to $\beta$.

Proof. When $\beta=[123]$, this is Proposition 5.3. Since the action of $\mathfrak{S}_{6}$

\begin{tabular}{|c|c|c|c|c|c|c|c|c|}
\hline \multirow[t]{2}{*}{$\beta$} & [123] & \multicolumn{2}{|c|}{ [124] } & \multicolumn{3}{|c|}{ [125] } & [126] & [134] \\
\hline & $e_{1}+f_{2}+g$ & $\mathrm{e}_{2}+$ & $f_{1}+g$ & $e_{1}+f_{1}$ & $e_{2}+f_{2}$ & $+g$ & g & $f_{1}+f_{2}+g$ \\
\hline & $\beta$ & [135] & {$[136]$} & [145] & [146] & & {$[156]$} & \\
\hline & & $+g$ & $e_{1}+g$ & $f_{2}+g$ & $\mathrm{e}_{2}+\mathrm{g}$ & & $e_{2}$ & \\
\hline
\end{tabular}
is compatible with the observation above, the general case follows.

By Proposition 5.4, we can write down the generator of the patching subgroup of the switch $\sigma_{\beta}$ for all $\beta$. We use the notations of Lemma 4.2.

Since all these are distinct each other, we see that the ten switches are not conjugate each other in $\operatorname{Aut}(X)$ if $X$ is Picard-general. 


\section{§6. Hutchinson's involutions associated with Göpel tetrads}

These automorphisms appear in [7]. The generic freeness is found by J. H. Keum in [10]. We briefly recall the construction. Let $G$ be a Göpel tetrad. If we choose $G$ as the reference points of the homogeneous coordinates of $\mathbb{P}^{3}$, the equation of $\bar{X}$ becomes

$$
\begin{aligned}
& A\left(x^{2} t^{2}+y^{2} z^{2}\right)+B\left(y^{2} t^{2}+z^{2} x^{2}\right)+C\left(z^{2} t^{2}+x^{2} y^{2}\right)+D x y z t \\
& \quad+E(y t+z x)(z t+x y)+G(z t+x y)(x t+y z)+H(x t+y z)(y t+z x)=0
\end{aligned}
$$

for suitable scalars $A, \ldots, H . \sigma_{G}$ is the Cremona involution

$$
(x, y, z, t) \longmapsto(1 / x, 1 / y, 1 / z, 1 / t) .
$$

For a translation $t=t_{\alpha}$, we have $\sigma_{t(G)}=t \sigma_{G} t$, so that we can restrict ourselves to the case $G$ is a Göpel subgroup. But any Göpel subgroup is of the form $\left\{n_{0}, n_{i j}, n_{k l}, n_{m n}\right\}$ hence up to $\mathfrak{S}_{6}$ we can assume $G_{0}=$ $\left\{n_{0}, n_{12}, n_{34}, n_{56}\right\}$. By [11], the induced action of $\sigma_{G_{0}}$ on $N S(X)$ is given by

$$
\begin{gathered}
N_{\alpha} \longleftrightarrow H-N_{0}-N_{12}-N_{34}-N_{56}+N_{\alpha}, \quad \text { for } \alpha=[0],[12],[34],[56] \\
T_{1} \longleftrightarrow T_{2}, T_{3} \longleftrightarrow T_{4}, T_{5} \longleftrightarrow T_{6}, \\
T_{134} \longleftrightarrow T_{234}, T_{123} \longleftrightarrow T_{124}, T_{125} \longleftrightarrow T_{126} .
\end{gathered}
$$

Proposition 6.1. The $(-1)$-eigenspace $K$ of $\sigma_{G_{0}}$ is generated over $\mathbb{Z}$ by the following elements.

$$
\begin{gathered}
g=N_{0}+N_{12}+N_{34}+N_{56}-H, \\
e_{5}=T_{1}-T_{2}, \quad e_{1}=T_{3}-T_{4}, \quad e_{7}=T_{5}-T_{6}, \\
f=T_{134}-T_{234}, \quad e_{3}=T_{123}-T_{124}, \quad h=T_{125}-T_{126}, \\
e_{2}=(1 / 2)\left(f+g+h-e_{3}\right), \quad e_{4}=(1 / 2)\left(f-e_{1}-e_{3}-e_{5}\right), \\
e_{6}=(1 / 2)\left(f+h-e_{5}-e_{7}\right) .
\end{gathered}
$$

Proof. $e_{1}, e_{3}, e_{5}, e_{7}, f, g, h \in K$ generate a sublattice of $K$ isomorphic to $A_{1}(2)^{\oplus 7}$. It is easy to see that $e_{2}, e_{4}, e_{6} \in N S(X)$. For example, modulo $N S(X)$,

$$
\begin{aligned}
e_{2} \equiv(1 / 2)\left(H+N_{0}+N_{12}+N_{34}+N_{56}\right. \\
\left.\quad+T_{123}+T_{124}+T_{125}+T_{126}+T_{134}+T_{234}\right) \\
=2 H+N_{0}-(1 / 2) \sum_{\alpha \in J(C)_{2}} N_{\alpha} .
\end{aligned}
$$


and as in Section $5 e_{2} \in N S(X) . e_{4}, e_{6}$ are similar.

Then we see that $e_{1}, \ldots, e_{7}$ span a lattice isomorphic to $E_{7}(2)$.

Proposition 6.2. The patching subgroup of $\sigma_{G_{0}}$ is 2-elementary abelian and generated by

$$
x=\left(N_{0}+N_{12}+N_{34}+N_{56}\right) / 2 \text {, and } y=H / 2 .
$$

Proof. This proposition is proved in the same way as Proposition 5.3. The corresponding element in $K^{*} / K$ is $x^{\prime}=\left(e_{1}+e_{3}\right) / 2, y^{\prime}=\left(e_{1}+e_{5}+e_{7}\right) / 2$ and we can check $x-x^{\prime}, y-y^{\prime} \in N S(X)$. Then we use Lemma 2.5.

By the $\mathfrak{S}_{6}$-symmetry, we obtain the following.

Proposition 6.3. For any Göpel subgroup $G$, we have $\Gamma_{\sigma_{G}}=\langle H / 2$, $\left.(1 / 2) \sum_{\alpha \in G} N_{\alpha}\right\rangle$.

More generally, using the translation relation $\sigma_{t(G)}=t \sigma_{G} t$, the generator above is valid for any Göpel tetrad.

There are 15 Göpel subgroups. Under the notations of Lemmas 4.1 and 4.2 , we deduce the following table.

\begin{tabular}{ll}
\hline The tetrad & Patching element corresponding to $x$ \\
\hline$[0]+[12]+[34]+[56]$ & $\mathrm{e}_{1}+\mathrm{f}_{1}+\mathrm{e}_{2}+\mathrm{f}_{2}$ \\
{$[0]+[12]+[35]+[46]$} & $\mathrm{f}_{1}+\mathrm{e}_{2}$ \\
{$[0]+[12]+[36]+[45]$} & $\mathrm{e}_{1}+\mathrm{f}_{2}$ \\
{$[0]+[13]+[24]+[56]$} & $\mathrm{e}_{1}+\mathrm{f}_{1}+2 \mathrm{~g}$ \\
{$[0]+[13]+[25]+[46]$} & $\mathrm{e}_{1}+\mathrm{f}_{1}+\mathrm{f}_{2}+2 \mathrm{~g}$ \\
{$[0]+[13]+[26]+[45]$} & $\mathrm{f}_{2}$ \\
{$[0]+[14]+[23]+[56]$} & $\mathrm{e}_{2}+\mathrm{f}_{2}+2 \mathrm{~g}$ \\
{$[0]+[14]+[25]+[36]$} & $\mathrm{f}_{1}+\mathrm{e}_{2}+\mathrm{f}_{2}+2 \mathrm{~g}$ \\
{$[0]+[14]+[26]+[35]$} & $\mathrm{f}_{1}$ \\
{$[0]+[15]+[23]+[46]$} & $\mathrm{e}_{1}+\mathrm{e}_{2}+\mathrm{f}_{2}+2 \mathrm{~g}$ \\
{$[0]+[15]+[24]+[36]$} & $\mathrm{e}_{1}+\mathrm{f}_{1}+\mathrm{e}_{2}+2 \mathrm{~g}$ \\
{$[0]+[15]+[26]+[34]$} & $\mathrm{f}_{1}+\mathrm{f}_{2}$ \\
{$[0]+[16]+[23]+[45]$} & $\mathrm{e}_{1}$ \\
{$[0]+[16]+[24]+[35]$} & $\mathrm{e}_{2}$ \\
{$[0]+[16]+[25]+[34]$} & $\mathrm{e}_{1}+\mathrm{e}_{2}$ \\
\hline
\end{tabular}

Since all these are distinct each other, we see that the 15 Hutchinson involutions are not conjugate each other in $\operatorname{Aut}(X)$ if $X$ is Picard-general. 
Remark. In [13] it is shown that if $(C, G)$ is bielliptic, then the involution $\sigma_{G}$ cannot be defined.

\section{§7. Hutchinson's involutions associated with Weber hexads}

These automorphisms appear in [5], [6]. The freeness is found in [4]. We fix a Weber hexad $W$. Then the linear system $L=\left|\mathcal{O}_{\bar{X}}(2)-W\right|$ with the assigned base points at $W$ defines an another quartic model $\bar{X}_{W}$ of $X$ in $\mathbb{P}^{4}$,

$$
\bar{X}_{W}: s_{1}+\cdots+s_{5}=0, \lambda_{1} / s_{1}+\cdots+\lambda_{5} / s_{5}=0,
$$

where $\lambda_{i}$ are nonzero constants.

$\sigma_{W}$ is the Cremona involution

$$
\sigma_{W}:\left(s_{1}, \ldots, s_{5}\right) \longmapsto\left(\lambda_{1} / s_{1}, \ldots, \lambda_{5} / s_{5}\right),
$$

preserving $\bar{X}_{W}$. It is free if $X$ is Picard-general [4]. For any translation $t=t_{\alpha}$, we have $\sigma_{t(W)}=t \sigma_{W} t$ as in the Hutchinson case. Hence we can assume that the Weber hexad doesn't contain $n_{0}$. Then recalling (4.1) in Section 4 , we have only one Weber hexad $W_{0}=\left\{n_{12}, n_{23}, n_{31}, n_{14}, n_{25}, n_{36}\right\}$ up to the action of $\mathfrak{S}_{6}$. In the following we discuss this case.

LEMMA 7.1. ([4]) $\sigma_{W_{0}}$ interchanges the following 10 pairs of smooth rational curves.

$$
\begin{aligned}
& \left(N_{0}, T_{123}\right), \quad\left(N_{56}, T_{1}\right), \quad\left(N_{46}, T_{2}\right), \quad\left(N_{45}, T_{3}\right), \quad\left(N_{15}, T_{124}\right), \\
& \left(N_{16}, T_{134}\right),\left(N_{24}, T_{125}\right),\left(N_{26}, T_{146}\right),\left(N_{34}, T_{136}\right), \quad\left(N_{35}, T_{236}\right) \text {. }
\end{aligned}
$$

Proposition 7.2. The $(-1)$-eigenspace $K$ of $\sigma_{W_{0}}$ is generated over $\mathbb{Z}$ by the following elements.

$$
\begin{gathered}
e_{1}=T_{2}-N_{46}, \quad e_{2}=N_{15}-T_{124}, \quad e_{3}=T_{1}-N_{56}, \\
e_{4}=N_{0}-T_{123}, \quad e_{5}=T_{3}-N_{45}, \quad e_{6}=N_{34}-T_{136}, \\
e_{7}=N_{23}-N_{56}-N_{34}-N_{24}-T_{134}-T_{124} .
\end{gathered}
$$

Proof. By computing the determinant, we can see that 10 divisors

$$
N_{0}+T_{123}, \ldots, N_{35}+T_{236}
$$

from Lemma 7.1 span over $\mathbb{Q}$ the invariant sublattice. $e_{1}, \ldots, e_{6} \in K$ is easy. $e_{7} \in K$ follows from the fact that $e_{7}$ is orthogonal to all of the divisors in (7.1). Then $e_{1}, \ldots, e_{7}$ spans the lattice $E_{7}(2) \simeq K$. 
Remark. The action of $\sigma_{W}$ on $N S(X)$ is very complicated, but essentially we can write down this action using the proposition above. In fact we find the following.

Let $W$ be a general Weber hexad. The "degree 1 part" $W_{1}$ of $W$ is the set

$$
\left\{\beta \in S(C) \mid\left(T_{\beta}, \sum_{\alpha \in W} N_{\alpha}\right)=1\right\} .
$$

$W_{1}$ consists of 6 elements. We have a natural bijection $\mu: W \rightarrow W_{1}$ defined by $\left(N_{\alpha}, T_{\mu(\alpha)}\right)=1$. On the other hand, for $\alpha \notin W$, we have the unique decomposition

$$
W=G \ominus R, \quad G \cap R=\left\{n_{\alpha}\right\} .
$$

Let $R^{\perp}$ be the 2-dimensional affine subspace of $J(C)_{2}$ which is orthogonal to $R$ and contains $n_{\alpha}$. Then $R \odot R^{\perp}$ is a Rosenhain hexad, i.e., $R \odot R^{\perp}$ is of the form $\Lambda(\beta)$ for some $\beta \in S(C)$. This defines a bijection $\mu^{\prime}: J(C)_{2}-W \rightarrow$ $S(C)-W_{1}, \alpha \mapsto \mu^{\prime}(\alpha)=\beta$. Using these data, the action of $\sigma_{W}$ is as follows.

$$
\begin{array}{rlrl}
\sigma_{W}\left(N_{\alpha}\right) & =3 H-\left(\sum_{\alpha \in J(C)_{2}} N_{\alpha}\right) / 2-\left(\sum_{\alpha \in W} N_{\alpha}\right)-T_{\mu(\alpha)}, & & \text { if } \alpha \in W . \\
\sigma_{W}\left(N_{\alpha}\right) & =T_{\mu^{\prime}(\alpha)}, & & \text { if } \alpha \notin W . \\
\sigma_{W}(H) & =9 H-\left(\sum_{\alpha \in J(C)_{2}} N_{\alpha}\right)-4\left(\sum_{\alpha \in W} N_{\alpha}\right) . &
\end{array}
$$

Proposition 7.3. The patching subgroup of $\sigma_{W_{0}}$ is cyclic and generated by

$$
x=(3 / 4) H+(1 / 2)\left(N_{12}+N_{23}+N_{31}+N_{14}+N_{25}+N_{36}\right) .
$$

Proof. The corresponding element in $K^{*} / K$ is

$$
y=\frac{1}{4} e_{1}+\frac{1}{2} e_{2}+\frac{1}{2} e_{4}+\frac{3}{4} e_{5}+\frac{1}{4} e_{7},
$$

and we check $x-y \in N S(X)$.

By the $\mathfrak{S}_{6}$-symmetry and the translation relation, we obtain

Proposition 7.4. For general $W$, the patching subgroup of $\sigma_{W}$ is

$$
\Gamma_{\sigma_{W}}=\left\langle(3 / 4) H+\left(\sum_{\alpha \in W} N_{\alpha}\right) / 2\right\rangle .
$$


There are 12 Weber hexads modulo translations. One more relation is hidden in the remark above. For $\alpha \notin W$, we have the unique decomposition $W=G \ominus R, G \cap R=\left\{n_{\alpha}\right\}$. Let $R^{\perp}$ be the orthogonal complement of $R$ at $n_{\alpha}$ and let $W_{\alpha}^{\perp}$ be the Weber hexad $G \odot R^{\perp}$. Then $\sigma_{W}$ and $\sigma_{W_{\alpha}^{\perp}}$ are conjugate, related by $\sigma_{W_{\alpha}^{\perp}}=\sigma_{\mu^{\prime}(\alpha)} \sigma_{W} \sigma_{\mu^{\prime}(\alpha)}$. Modulo this relation, we have 6 Weber hexads. Under the notations of Lemmas 4.1 and 4.2, their patching subgroups are as follows.

\begin{tabular}{ll}
\hline Weber hexad & patchings \\
\hline$[12]+[23]+[31]+[14]+[25]+[36]$ & $\mathrm{e}_{1}+\mathrm{f}_{1}+\mathrm{e}_{2}+\mathrm{g}$ \\
{$[12]+[13]+[23]+[24]+[15]+[36]$} & $\mathrm{f}_{1}+\mathrm{e}_{2}+\mathrm{f}_{2}+\mathrm{g}$ \\
{$[23]+[13]+[12]+[34]+[25]+[16]$} & $\mathrm{e}_{2}+\mathrm{f}_{2}+\mathrm{g}$ \\
{$[24]+[23]+[34]+[14]+[25]+[36]$} & $\mathrm{e}_{1}+\mathrm{f}_{1}+\mathrm{f}_{2}+\mathrm{g}$ \\
{$[25]+[23]+[35]+[54]+[21]+[36]$} & $\mathrm{e}_{1}+\mathrm{e}_{2}+\mathrm{f}_{2}+\mathrm{g}$ \\
{$[26]+[23]+[36]+[64]+[25]+[13]$} & $\mathrm{e}_{1}+\mathrm{f}_{1}+\mathrm{g}$
\end{tabular}

Thus we see that there are $6 \mathrm{HW}$ involutions that are not conjugate each other in $\operatorname{Aut}(X)$ if $X$ is Picard-general.

Remark. (1) In a forthcoming paper we will be able to determine when $\sigma_{W}$ is not free.

(2) The 6 conjugacy classes of HW involutions are naturally "dual" to the 6 Weierstrass points, in the sense that the $\mathfrak{S}_{6}$ action on both factors through an outer automorphism. Details are as follows. There are 20 Weber hexads $W$ which don't contain $n_{0}$ and conjugate each other. Writing $W$ uniquely as $W=G \ominus R$ with $G \cap R=\left\{n_{0}\right\}$, we can associate with such $W$ the Göpel subgroup $G$. But a Göpel subgroup $G=\left\{n_{0}, n_{i j}, n_{k l}, n_{m n}\right\}$ is determined just by the "syntheme" $(i j)(k l)(m n) \in \mathfrak{S}_{6}$. Thus we obtain 20 synthemes from the conjugacy class. The fact is that there appear only 10 synthemes, and the synthemes not appearing here form a "total", which is the classical description of the dual of the 6 -set $\{1, \ldots, 6\}$.

(3) The method of this paper is applicable to the case of Picard-general quartic Hessian surfaces of [4]. In this case we have exactly one Enriques quotient.

Proof of Theorem 1.2. Let $N^{\prime}$ be the group generated by 16 translations $t_{\alpha}, 16$ switches $\sigma_{\beta}, 16$ projections $p_{\alpha}, 16$ correlations $p_{\beta}, 60 \mathrm{HG}$ involutions $\sigma_{G}, 192 \mathrm{HW}$ involutions $\sigma_{W}$. The theorem follows from the following lemma as in [12, Lemma 7.3]. 
LEMMA 7.5. Let $\phi$ be an isometry of $N S(X)$ that preserves the ample cone. Then there exists a $g \in N^{\prime}$ such that $g \phi \in \operatorname{Aut}\left(D^{\prime}\right)$.

Proof. Let $w^{\prime}=2 H-\sum N_{\alpha} / 2$ be the projection of the Weyl vector $w$. Let $v=\phi\left(w^{\prime}\right)$ and let $g \in N^{\prime}$ be an element that attains the minimum $\min \left\{\left(g(v), w^{\prime}\right) \mid g \in N^{\prime}\right\}$. If $r$ is the Leech root corresponding to [12, Lemma 4.6, Case (0), (1), (2)], then as in [12] we have $\left(r^{\prime}, g(v)\right)>0$.

If $r$ is the Leech root corresponding to [12, Lemma 4.6, Case (3)], then it corresponds to some Weber hexad $W$ and [12, Remark 6.3, (1)] can be rewritten as

$$
4 r^{\prime}=3 H-2 \sum_{\alpha \in W} N_{\alpha} .
$$

Using Proposition 7.2 and its Remark, we have

$$
\sigma_{W}\left(w^{\prime}\right)=w^{\prime}+8 r^{\prime}
$$

Thus, we have

$$
\begin{aligned}
& \left(g(v), w^{\prime}\right) \leq\left(g(v), \sigma_{W}^{-1}\left(w^{\prime}\right)\right)=\left(g(v), w^{\prime}\right)+8\left(g(v), r^{\prime}\right), \\
& \left(g(v), r^{\prime}\right)>0 .
\end{aligned}
$$

Hence $g(v) \in D^{\prime}$.

Remark. Unfortunately, $\operatorname{Aut}(X)$ cannot be generated only by the subset

$$
\begin{array}{r}
S=\left\{t_{\alpha}, \sigma_{\beta}, \sigma_{G}, \sigma_{W} \mid \alpha \in J(C)_{2}, \beta \in S(C),\right. \\
G: \text { Göpel tetrad, } W: \text { Weber hexad }\},
\end{array}
$$

introduced in this paper. It is easy to see that for any element $\varphi$ written as a product of elements in $S$, we have $\left(w^{\prime}, \varphi\left(w^{\prime}\right)\right) \in 4 \mathbb{Z}$. But the projection $p_{\alpha}$ have $\left(w^{\prime}, p_{\alpha}\left(w^{\prime}\right)\right)=26$.

Acknowledgement. The author expresses his sincere gratitude to Professor Shigeru Mukai. He suggested using Torelli theorem for Enriques surfaces in proving Proposition 2.1, which was a better method of counting than that of [16] and an important step for the computation in this paper. He explained his study of [13] in process and led the author to his conjecture.

Financial support has been provided by the Research Fellowships of the Japan Society for the Promotion of Science for Young Scientists. 


\section{REFERENCES}

[1] W. Barth and C. Peters, Automorphisms of Enriques surfaces, Invent. math., 73 (1983), 383-411.

[2] N. Bourbaki, Éléments de Mathématique. Groupes et Algèbres de Lie, Chap. IV, V et VI.

[3] J. H. Conway and N. J. A. Sloane, Sphere packings, lattices and groups, 3rd ed., Grundlehren der Mathematischen Wissenschaften 290, Springer-Verlag, 1999.

[4] I. V. Dolgachev and J. H. Keum, Birational automorphisms of quartic hessian surfaces, Trans. Amer. Math. Soc., 354 (2002), 3031-3057.

[5] J. I. Hutchinson, The Hessian of the cubic surface, Bull. Amer. Math. Soc., 5 (1899), $282-292$.

[6] J. I. Hutchinson, The Hessian of the cubic surface, II, Bull. Amer. Math. Soc., 6 (1899), 328-337.

[7] J. I. Hutchinson, On some birational transformations of the Kummer surfaces into itself, Bull. Amer. Math. Soc. (2), 7 (1901), 211-217.

[8] F. Klein, Ueber Configurationen, Welche der Kummer'schen Fläche Zugleich Eingeschrieben und Umgeschrieben Sind, Math. Ann., 27 (1886), 106-142.

[9] M. R. Gonzalez-Dorrego, $(16,6)$ configurations and geometry of Kummer surfaces in $\mathbb{P}^{3}$, Mem. Amer. Math. Soc. 107, 1994.

[10] J. H. Keum, Every algebraic Kummer surface is the K3-cover of an Enriques surface, Nagoya Math. J., 118 (1990), 99-110.

[11] J. H. Keum, Automorphisms of Jacobian Kummer surfaces, Compositio Math., 107 (1997), 269-288.

[12] S. Kondo, The automorphism group of a generic Jacobian Kummer surface, J. Algebraic Geometry, 7 (1998), 589-609.

[13] S. Mukai, Kummer's quartics and numerically reflective involutions of Enriques surfaces, RIMS Preprint, 1633.

[14] V. V. Nikulin, An analogue of the Torelli theorem for Kummer surfaces of Jacobians (English translation), Math. USSR Izv., 8 (1974), 21-41.

[15] V. V. Nikulin, Integral symmetric bilinear forms and some of their applications (English translation), Math. USSR Izv., 14 (1980), 103-167.

[16] H. Ohashi, On the number of Enriques quotients of a K3 surface, Publ. Res. Inst. Math. Sci., 43 (2007), 181-200.

[17] H. Ohashi, Counting Enriques quotients of a K3 surface, RIMS Preprint, 1609.

Research Institute for Mathematical Sciences

Kyoto University

Sakyo-ku, Kyoto 606-8502

Japan

pioggia@kurims.kyoto-u.ac.jp 